\title{
An evaluation of the use of the South African Triage Scale in an urban district hospital in Durban, South Africa
}

\author{
S Sooguna* iD, M Naidoo and K Naidoo ${ }^{a}$ \\ aDiscipline of Family Medicine, School of Nursing and Public Health, University of KwaZulu-Natal, Durban, South Africa \\ *Corresponding author, email: sunsho@yahoo.com
}

Background: Emergency centres in South Africa are among the busiest in the world and serve as entry points for hospital care for most of the population. The South African Triage Scale (SATS) is a validated tool introduced nationally in 2006 and intended to increase the efficiency of emergency centres through a process of prioritisation of the severely ill patient. The objective of this study was to evaluate the use of the SATS in a busy urban district hospital in Durban, South Africa.

Methods: A chart review of triaged patients was performed. The hospital uses a one-page SATS sheet and manages both medical and surgical patients. The triage history, physiological parameters, application of discriminator lists, final triage code and outcome was audited and compared with findings from the patients' clinical records.

Results: The mean triage early warning score was 1.50 (95\% Cl 1-2) and average time to treatment was 59 min $(95 \% \mathrm{Cl} 51-$ 67). Essential bedside investigations were missing on some very ill patients, there was poor documentation in many fields and confirmed time to treatment was within recommended timescales for only $48 \%$ of patients. Use of the discriminator list resulted in over-triage of $66.7 \%$ and an under-triage of $14 \%$. Some $76 \%$ of patients were discharged from the emergency centre, $15 \%$ were admitted and $5.5 \%$ were transferred out.

Conclusion: Nurse-led triage has been successfully implemented at the emergency centre of this hospital using SATS but some notable gaps were identified.

Keywords: audit, emergency centre, South African Triage Scale

\begin{abstract}
Background
Emergency centres in South Africa are very busy, often flooded by a trauma load that is one of the highest in the world, chronic medical conditions, acute medical emergencies and HIV-related conditions. ${ }^{1-4}$ The government hospitals cater for the majority of the population but these hospitals are poorly funded, ill equipped and often do not have skilled staff. 1,4,5 The district hospitals, which are level one hospitals, often serve as the entry points for hospital care for patients referred by local clinics, private general practitioners and those that self-refer. In such a setting, it is important to have a reliable method of sorting or triaging patients so that the severely ill patient who is at risk of deterioration is promptly identified and treated. This was the reason for the introduction, in 2006, of the South African Triage Scale (SATS) in South Africa and its adoption in many hospitals in Africa and some low- and middle-income Asian countries. ${ }^{2,6,7}$
\end{abstract}

The SATS consists of three elements involving the documentation of the Triage Early Warning Score (TEWS), the discriminator list, and the final opinion of a senor healthcare professional. ${ }^{2,4,8}$ The patient's vital signs are measured and scores are allocated. There is also a score for mobility and level of consciousness. The scores are added at the end of the process and the total score is documented. The discriminator list is a set of emergency (red), very urgent (orange), and urgent (yellow) conditions that places a patient in the corresponding category irrespective of the TEWS. The third part of the SATS allows for an experienced senior healthcare professional to provide oversight of the process. ${ }^{9,10}$ The SATS is specially designed for use in low-resource clinical settings. It is also simple, easy to use and can be used effectively by nursing assistants in emergency centres providing care to both medical and surgical patients. ${ }^{2,8}$ It improves the efficiency of emergency centres, reduces patients' waiting times, improves patient flow and improves job satisfaction among staff. ${ }^{1,9,11}$ However, these benefits are dependent on the correct implementation of the tool.

Previous studies that support and validate the use of the SATS were conducted mainly in large secondary- and tertiary-level hospitals situated in either urban or rural parts of South Africa. ${ }^{1,11-13}$ These hospitals are often better staffed and equipped than district hospitals. A previous audit on the use of SATS in the National District Hospital in Bloemfontein concluded that the tool was poorly implemented. ${ }^{3}$ The SATS was introduced at the emergency centre of the Wentworth Hospital, Durban in 2008. Since introduction, its use at this hospital has not been evaluated. The aim of this study is to evaluate the use of the SATS in this busy urban district hospital in Durban, South Africa.

The specific objectives of the study were to determine whether all components of the TEWS were done and recorded correctly, if all patients were colour coded, if the time to treatment of patients was within the recommended time frames for specific colour codes, the level of over-triage and under-triage, and to describe patterns in the quality of triage during working hours and after hours.

\section{Methods}

A chart review was conducted at this 246-bed urban district hospital in Durban, South Africa which provides a 24-hour emergency service. The hospital uses a one-page SATS sheet that is added to the patient's clinical record. Triage is done by the nursing staff working at the hospital who take a brief history, record the vital signs, use the discriminator list provided on the SATS sheet and then triage patients into red (emergency), orange (urgent), yellow (semi-urgent), green (not urgent) or blue (dead 
on arrival) codes. The hospital serves a catchment population of 334000 people and the emergency centre sees an average of 2 200 patients per month.

A sample size of over 300 records from a finite population of 2 200 per month produces a two-sided $95 \%$ confidence interval with a precision (half-width) of \pm 0.05 or $\pm 5 \%$ when the actual proportion is near 0.50 or $50 \%$ (assumes maximum variability given no local prior estimates to factor into the sample size calculation) The actual number of patients seen in the month of December 2015 was 2 128. The sample size of 346 was arrived at after randomly choosing 10 days of the month of December 2015. The dates for the review were finalised using an online random number generator. Clinical records for all patients older than 12 years of age were included in the study. We excluded files of children less than 12 years of age, patients who were referred from other departments to review investigations and files in which the triage sheet was missing.

Data were obtained, in January 2016, by extracting information from patients' files using a data-collection tool. The datacollection tool was based on the study objectives and was first piloted and modified before final use. Variables extracted were based on the gender of the patient, the triage history, the time of triage, patients' vital signs, the side-room tests performed, application of the discriminator lists, the opinion of the senior healthcare worker, the colour coding of the patient, the time taken to see the doctor and the patient outcome. The findings in the triage sheet were compared with the clinical notes in the patient's file.

The data collected were entered into SPSS version 23@ (IBM Corp, Armonk, NY, USA) and analysed. Ethical approval was obtained from the Biomedical Research Ethics Committee of the University of KwaZulu-Natal (Reference BE330/15). Gatekeeper permission was obtained from the KZN provincial Department of Health and the hospital manager. Patient identifiers were protected by assigning a unique study identification number for each file captured.

\section{Results}

Of the 346 files reviewed, 201 were male $(58.1 \%)$ and 145 female (41.9\%) with 140 patients being seen during working hours (40.5\%), 201 after hours (58.1\%) and the time of day could not be determined in 5 cases (1.5\%). The mean TEWS score was 1.50 $(95 \% \mathrm{Cl} 1 ; 2)$ indicating that most patients were coded as green codes. The average time to treatment is $59 \min (95 \% \mathrm{Cl} 51 ; 67)$. Table 1 is an evaluation of the triage sheet when information on the triage sheet was compared with that in the patient's clinical notes.

Most parameters were correctly recorded except for the trauma score. The total TEWS was incorrectly or not recorded in 64 $(18.4 \%)$ cases and the final triage score was incorrectly or not recorded in 84 (24.3\%) cases.

The complimentary bedside investigations done during triage included blood glucose estimation, oxygen saturation, urine dipstick and a urine pregnancy test when indicated. Blood glucose was done in 335 cases (96.8\%), Oxygen saturation was measured in 339 patients (98\%). The urine dipstick was done in $99(28.6 \%)$ cases. Urine pregnancy test was done in 59 (17.1\%) cases, not done in $75(21.7 \%)$ cases and was not applicable in 212 $(61.3 \%)$ cases. Table 2 cross-tabulates bedside investigations with the final triage colour.
Table 1: Comparison of the triage history and TEWS with patients' clinical notes

\begin{tabular}{lccc}
\hline Factor & $\begin{array}{c}\text { Recorded } \\
\text { correctly (\%) }\end{array}$ & $\begin{array}{c}\text { Recorded } \\
\text { incorrectly (\%) }\end{array}$ & $\begin{array}{c}\text { Not recorded } \\
(\%)\end{array}$ \\
\hline Triage history & $339(98.0)$ & $1(0.3)$ & $6(1.7)$ \\
\hline Mobility & $342(98.8)$ & 0 & $4(1.2)$ \\
\hline Respiration & $344(99.4)$ & 0 & $2(0.6)$ \\
\hline Heart rate & $343(99.1)$ & $1(0.3)$ & $2(0.6)$ \\
\hline Blood pressure & $344(99.4)$ & $1(0.3)$ & $1(0.3)$ \\
\hline Temperature & $342(98.8)$ & $0(0.0)$ & $4(1.2)$ \\
\hline AVPU & $340(98.3)$ & $1(0.3)$ & $5(1.4)$ \\
\hline Trauma & $323(93.4)$ & $16(4.6)$ & $7(2.0)$ \\
\hline Total TEWS & $282(81.5)$ & $12(3.4)$ & $52(15.0)$ \\
\hline Final triage & $262(75.7)$ & $35(10.1)$ & $49(14.2)$ \\
\hline
\end{tabular}

Notes: AVPU: Alert, responsive to verbal command, responds to pain, unresponsive. Percentages of the total sample recorded in parentheses. Total number of patients $=346$.

Table 2: Bedside tests cross-tabulated with the final triage colour

\begin{tabular}{lccccc}
\hline $\begin{array}{l}\text { Bedside } \\
\text { test }\end{array}$ & \multicolumn{5}{c}{ Final triage colour code (\%) } \\
\cline { 2 - 5 } & Red (4) & $\begin{array}{c}\text { Orange } \\
(\mathbf{5 6})\end{array}$ & $\begin{array}{c}\text { Yellow } \\
\mathbf{( 7 3 )}\end{array}$ & $\begin{array}{c}\text { Green } \\
(\mathbf{1 6 4 )}\end{array}$ & $\begin{array}{c}\text { Not } \\
\text { coded } \\
(49)\end{array}$ \\
\hline $\begin{array}{l}\text { Blood } \\
\text { glucose }\end{array}$ & 4 & $55(98.2)$ & $69(94.5)$ & $159(97.0)$ & $48(98)$ \\
$\begin{array}{l}\text { Oxygen } \\
\text { saturation }\end{array}$ & 4 & $55(98.2)$ & $71(97.2)$ & $160(97.6)$ & $49(100)$ \\
$\begin{array}{l}\text { Urine } \\
\text { dipstick }\end{array}$ & 0 & $19(33.9)$ & $26(35.6)$ & $37(22.6)$ & $17(94.7)$ \\
$\begin{array}{l}\text { Pregnancy } \\
\text { test }\end{array}$ & 0 & $11(19.6)$ & $16(21.9)$ & $22(13.4)$ & $9(18.4)$ \\
\hline
\end{tabular}

Note: Percentage is of the total number per colour category.

No red-coded patient had a urine dipstick test done and this bedside test was done in the minority of patients coded orange, yellow and green. Almost all patients had blood sugar and oxygen saturation levels performed. The bedside investigations often help in the application of the discriminator list. The discriminator list was used to up-triage patients accurately in $27.7 \%$ of cases, inaccurately applied in four (1.7\%) cases and not needed in the rest. The senior health professional input was used to clarify triage colour in 26 (7.5\%) of the cases reviewed.

The final colour coding of patients as documented in the patients' triage charts were compared with the outcome and the time to treatment. The time to treatment is the time difference between the time the patient was triaged and the time the patient was seen by the doctor. Table 3 cross-tabulates the final triage colour with the eventual outcome and the time to treatment.

One of the red code patients was discharged and three were admitted. Two hundred and sixty-three $(76 \%)$ patients were discharged, 52 (15\%) were admitted, 19 (5.5\%) were transferred to another facility and $10(2.9 \%)$ charts had no documented outcome. A total of 49 charts were not colour coded. One red code was seen immediately after triage and one red code was seen more than 60 minutes after triage. One hundred and seventy-one patients (49.4\%) were seen within one hour. Overtriage was $66.7 \%$ and under triage was $14 \%$. 
Table 3: Final triage colour cross-tabulated with outcome and time to treatment

\begin{tabular}{|c|c|c|c|c|c|c|}
\hline Factor & Red & Orange & Yellow & Green & $\begin{array}{l}\text { Not } \\
\text { coded }\end{array}$ & Total \\
\hline Frequency & $4(1.2)$ & $56(16.2)$ & $73(21.1)$ & $164(47.4)$ & 49 (14.2) & $346(100)$ \\
\hline \multicolumn{7}{|l|}{ Outcome } \\
\hline Discharged & 1 & $39(69.6)$ & $53(72.6)$ & $135(82.3)$ & 35 (71.4) & $263(76)$ \\
\hline Admitted & 3 & $11(19.6)$ & $12(16.4)$ & $17(10.4)$ & $9(18.4)$ & $52(15)$ \\
\hline Transfer & 0 & $5(8.9)$ & $5(6.8)$ & $6(3.7)$ & $3(6.1)$ & $19(5.5)$ \\
\hline Absconded & 0 & $0(0.0)$ & $1(1.4)$ & $0(0.0)$ & $1(2.0)$ & $2(0.6)$ \\
\hline $\begin{array}{l}\text { Not docu- } \\
\text { mented }\end{array}$ & 0 & $1(1.8)$ & $2(2.7)$ & $6(3.7)$ & $1(2.0)$ & $10(2.9)$ \\
\hline \multicolumn{7}{|c|}{ Time to treatment } \\
\hline $\begin{array}{l}\text { Immedi- } \\
\text { ately }\end{array}$ & 1 & 0 & 0 & 0 & 0 & $1(0.3)$ \\
\hline $1-10 \min$ & 0 & $12(21.4)$ & $9(12.3)$ & $17(10.4)$ & $7(14.3)$ & $45(13)$ \\
\hline $11-60 \mathrm{~min}$ & 2 & $24(42.9)$ & $28(38.4)$ & $63(38.4)$ & $11(22.4)$ & $128(37)$ \\
\hline $61-240 \min$ & 1 & $12(21.4)$ & $12(16.4)$ & $36(22)$ & $10(20.4)$ & $71(20.5)$ \\
\hline $\begin{array}{l}\text { More than } \\
240 \text { min }\end{array}$ & 0 & $0(0.0)$ & $3(4.1)$ & $2(1.2)$ & 0 & $5(1.5)$ \\
\hline $\begin{array}{l}\text { Not docu- } \\
\text { mented }\end{array}$ & 0 & $8(14.3)$ & $21(28.8)$ & $46(28)$ & 21 (42.9) & $96(27.7)$ \\
\hline
\end{tabular}

Note: Percentages (in parentheses) for outcome and time to treatment are of the total of each colour code.

Table 4: Time of day seen cross-tabulated with final triage colour, recommended time to treatment and outcome

\begin{tabular}{|c|c|c|c|c|}
\hline Factor & $\begin{array}{c}\text { Normal } \\
\text { working } \\
\text { hours (\%) }\end{array}$ & $\begin{array}{l}\text { After } \\
\text { hours } \\
(\%)\end{array}$ & $\begin{array}{c}\text { Not } \\
\text { documented } \\
(\%)\end{array}$ & $\begin{array}{l}\text { Total } \\
(\%)\end{array}$ \\
\hline \multicolumn{5}{|l|}{ Final triage colour } \\
\hline Red & $1(0.7)$ & $3(1.5)$ & 0 & $4(1.2)$ \\
\hline Orange & $19(13.6)$ & $37(18.4)$ & 0 & $56(16.2)$ \\
\hline Yellow & $30(21.4)$ & $42(20.9)$ & 1 & $73(21.1)$ \\
\hline Green & $70(50.0)$ & $91(45.3)$ & 3 & $164(47.4)$ \\
\hline Not coded & $20(14.3)$ & $28(13.9)$ & 1 & $49(14.2)$ \\
\hline \multicolumn{5}{|l|}{ Time to treatment } \\
\hline Within recommended & $68(48.6)$ & $98(48.8)$ & 0 & $166(48)$ \\
\hline $\begin{array}{l}\text { Outside recom- } \\
\text { mended }\end{array}$ & $22(15.7)$ & $34(16.9)$ & 0 & $56(16.2)$ \\
\hline $\begin{array}{l}\text { Could not be deter- } \\
\text { mined }\end{array}$ & $50(35.7)$ & $69(34.3)$ & 5 & $124(35.8)$ \\
\hline \multicolumn{5}{|l|}{ Outcome } \\
\hline Discharged & $105(75)$ & $\begin{array}{c}155 \\
(77.1)\end{array}$ & 3 & $263(76)$ \\
\hline Admitted & $22(15.7)$ & $30(14.9)$ & 0 & $52(15)$ \\
\hline Transferred & $10(7.1)$ & $8(4.0)$ & 1 & $19(5.5)$ \\
\hline Absconded & 0 & $1(0.5)$ & 1 & $2(0.6)$ \\
\hline Not documented & $3(2.1)$ & $7(3.5)$ & 0 & $10(2.9)$ \\
\hline
\end{tabular}

Note: Percentages shown in parentheses are of patients seen based on the time of day.

The odds of being admitted if you were a red code patient was $17.9(95 \% \mathrm{Cl} 1.4-941.4 ; p=0.01), 1.7(95 \% \mathrm{Cl} 0.7-3.7 ; p=0.3)$ if you were a patient coded as orange, $1.4(95 \% \mathrm{Cl} 0.6-2.9 ; p=0.7)$ if you were coded as yellow and $0.5(95 \% \mathrm{Cl} 0.3-0.9 ; p=0.02)$ if you were coded as green. Only the patients coded as red codes had significant odds of being admitted to hospital and patients coded as green had significant odds of not being admitted.

The time of day that patients were triaged were then crosstabulated to the triage codes, time to treatment and outcomes and this is shown in Table 4.

Recommended time is colour code dependent and is immediately for red, up to $10 \mathrm{~min}$ for orange, up to 60 minutes for yellow and up to 240 minutes for green. One hundred and sixty-six (48\%) patients were seen within the recommended time. Sixty (17.3\%) patients seen during the day or after hours could be emergencies or urgencies. The outcomes of the patients seen during working and after hours were similar in terms of the proportion of patients seen. The time to treatment could not be related to colour code in $124(35.8 \%)$ cases.

\section{Discussion}

Nurse-led triage has been successfully implemented at the emergency centre of the hospital using the SATS but there were some gaps identified. These gaps included the following: essential bedside investigations were missing on some very ill patients, there was poor documentation in many fields and we could only confirm that the time to treatment was within the recommended timescales for $48 \%$ of patients.

Patients who presented to the emergency centre directly were triaged using the age-appropriate SATS tool. More males were seen at Wentworth hospital emergency centre than females, which is consistent with findings in district hospitals in Paarl and Limpopo in South Africa., ${ }^{3,14}$ but different from findings in hospitals that do not see trauma cases, like the National District hospital in Bloemfontein, as males tend to present more often with trauma-related injuries than females. ${ }^{3,5,15}$ The mean TEWS suggests most cases seen were green codes, which is consistent with findings of the study done in Paarl where $81.8 \%$ were yellow and green codes. ${ }^{14}$ This is also consistent with the findings of Becker and colleagues, ${ }^{16}$ who in 2012 found that the majority of patients attending a secondary level hospital's emergency centre had primary care complaints due to their dissatisfaction with attending primary health centres. ${ }^{16}$ Our findings were different from findings from larger secondary and tertiary hospitals where the majority of cases were coded as yellow. ${ }^{12,17}$

The trauma score was added to TEWS to increase the sensitivity of the SATS score in identifying patients at risk of deterioration and to simplify the discriminator list for use in emergency centres providing care for medical and surgical patients. ${ }^{4}$ In our study, we observed that the trauma score was documented incorrectly more than the other components of the TEWS. This is probably because the trauma score was omitted by the triage nurse in patients with minor injuries. Specific incidents included patients who fell at home or were assaulted (including stab injuries) but were given a trauma score of zero while some healthcare workers omitted reporting on this parameter. This undermines the importance of the triage as a reliable, reproducible process devoid of staff error. Naidoo and colleague, ${ }^{13}$ who performed an evaluation at Addington hospital in Durban, found that $55.3 \%$ of charts had insufficient records for analysis, which is much higher than our study. ${ }^{13}$ Triage information was available in 1147 of 2134 cases analysed in Paarl. ${ }^{14}$ Bloemfontein reported $98 \%$ completion of physiological parameters in TEWS. ${ }^{3}$ None of the other studies reviewed outlined compliance with individual components of the TEWS. Missing information has the potential to compromise the quality of care and could lead to medico-legal problems. 
Bedside investigations are supposed to complement the TEWS to identify high-risk patients and apply an appropriate discriminator to up-triage the patient. These are probably not understood by the triage nurses. When the clinical notes were audited and compared with the triage history it was found that the history was very superficial and not goal oriented. Further training of the nurses performing the triage is needed to improve their skills so that they can identify red flags of urgent conditions, which will encourage focused bedside tests and application of the appropriate discriminator. An example in this study was that of a young woman with lower abdominal pain who was triaged as yellow (abdominal pain) instead of orange (abdominal pain in pregnancy). In this instance, the pregnancy test was requested by the medical officer only after the clinical examination. This scenario has the potential for disaster as conditions such as ruptured ectopic pregnancies are often missed or not managed with the urgency that these cases deserve, resulting in preventable mortality. ${ }^{18}$ Urine dipstick tests were omitted in some patients with hyperglycaemia, which may have resulted in a diabetic emergency being missed. Red code patients with a high TEWS score due to unstable vital signs require proper evaluation to identify the underlying pathology. This evaluation reasonably includes urine dipstick, which was omitted in all the red codes. Urine dipstick can help in organ system evaluation in very ill patients.

The discriminator list was applied to up-triage patients in $27.1 \%$ of cases, similar to findings of Gottschalk and colleagues ${ }^{6}$ who observed that discriminator lists improved triage priority in $23 \%$ of cases. Though sometimes said to be cumbersome and difficult to use by some researchers, the application of the discriminator list was meant to improve triage colour coding and medical intervention. The discriminator list can sometimes over-triage patients, which may account for the large number of orange codes being discharged.

Over-triage occurs when prioritised patients do not reach the expected outcome as predicted by the colour code; examples are red and orange codes that are discharged. Similarly, under-triage describes low-priority cases (green) that reached unexpected outcomes (admitted, death or transfer for higher care). In this study, over-triage was $66.7 \%$ and under-triage was $14 \%$. This is similar to over-triage of $67.9 \%$ and different from the under-triage of $0.3 \%$ found in Bloemfontein. ${ }^{3}$ The American College of Surgeons Committee on Trauma suggest that an over-triage rate of up to $50 \%$ and an under-triage rate of less than $5 \%$ is acceptable..$^{1,19}$ However, in resource-constrained settings with less skilled healthcare workers doing triage, a higher level of over- and undertriage would be expected. The senior healthcare professional made decisions on the final triage score in $7.5 \%$ of cases. The most common of such cases included bites, especially snake and dog bites in which the triage nurse was uncertain what to do and sought the opinion of the medical officer on duty. The medical officer often ended up examining and managing the patient within 10 minutes despite the patient having a low TEWS, which would have coded them as green cases.

Forty-eight percent of cases in our study were seen within the recommended time limits, which is higher than the $8 \%$ reported in the study done in Bloemfontein. ${ }^{3}$ The average time to treatment of 59 minutes in this study is within the national Department of Health target of 120 minutes. ${ }^{20}$ It could only be established that $16.2 \%$ of cases were seen outside the recommended time but this data item should be interpreted with caution due to the large percentage of undocumented times. This is worrying, in the context of escalating medical litigation in the public sector. ${ }^{21}$
The patient outcomes in this study were similar to those done in Bloemfontein and Paarl, which had an admission rate of $20.7 \%$ and $16.5 \%$ respectively. ${ }^{3,14}$ The discharge rate in Bloemfontein was $69.9 \%$, which is similar to our finding. ${ }^{14}$ Relatively higher admission rates of $40 \%$ at Ngwelezana hospital and $48.7 \%$ at Addington hospital were documented in the emergency centres, as these are bigger regional hospitals. ${ }^{1,13}$ One patient with a red code was discharged from the emergency centre and $75 \%$ of red codes were admitted, which is higher than the $37 \%$ admission rate for red-coded patients in Bloemfontein. ${ }^{3}$ The discharged patient had a TEWS score of eight indicating unstable vital signs and was seen by a doctor 160 minutes after triage and then surprisingly discharged without any documented evidence of improved vital signs. This is a serious concern as this has grave implications for patient safety and the threat of medical litigation.

There are similarities in the proportion of patients' outcomes, missing data, unrecorded TEWS and waiting times during working hours and after hours in other studies. ${ }^{3}$

The limitations of this study may be accounted for based on the retrospective nature of the study design and this may have accounted for the information bias. The missing data limited some aspects of the data analysis so appropriate conclusions could not be made. However, the strength of the study was the evaluation of an operational programme that avoided the Hawthorne effect.

In conclusion, the study evaluated the use of the SATS in this urban district hospital and found that nurse-led triage was successfully implemented but with some deficiencies. The main component of the TEWS that was poorly implemented is the recording of the trauma score, resulting in the incorrect calculation of the final TEWS score. Patients were appropriately colour coded in most cases but there were some instances in which the discriminator was not appropriately applied. Specific times at which patients were seen was often missing resulting in gaps in establishing the time to treatment. There was no significant difference between triage performed during office hours and after hours. To deal with this, feedback will be provided to medical and nursing staff at the hospital and focused training of nurses and doctors will be suggested. A quality improvement project needs to be implemented and the quality assurance manager needs to take responsibility for ongoing monitoring and evaluation. Informal feedback was provided to the interns working at the hospital and interns developed a strategy to improve the time to treatment, resulting in improved waiting times for patients coded as red or orange. Further research is recommended to evaluate the implementation of SATS in emergency centres in South Africa.

Acknowledgements - The authors would like to thank the nursing and administrative staff of Wentworth Hospital for assisting with the study.

Conflict of interest - The authors declare that no conflict of interests exists in this study.

\section{ORCID}

S Soogun (iD http://orcid.org/0000-0002-0812-2024

\section{References}

1. Rosedale K, Smith ZA, Davies H, et al. The effectiveness of the South African Triage Score (SATS) in a rural emergency department. S Afr Med J. 2011;101(8): 537-40. 
2. Gottschalk SB, Wood D, DeVries $S$, et al. The cape triage score: a new triage system South Africa. Proposal from the cape triage group. Emerg Med J. 2006;23(2): 149-153. https://doi.org/10.1136/ emj.2005.028332

3. Gordon S, Brits H, Raubenheimer J. The effectiveness of the implementation of the Cape Triage Score at the emergency department of the National District Hospital, Bloemfontein. South Afr Family Practice. 2015;57(1): 18-23. https://doi.org/10.1080/20786190 .2014 .977056

4. Bruijns SR, Wallis LA, Burch VC. A prospective evaluation of the Cape triage score in the emergency department of an urban public hospital in South Africa. Emerg Med J. 2008;25(7): 398-402. https:// doi.org/10.1136/emj.2007.051177

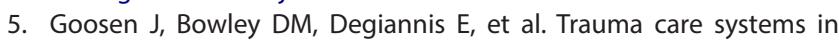
South Africa. Injury. 2003;34(9): 704-708. https://doi.org/10.1016/ S0020-1383(03)00153-0

6. Gottschalk SB, Warner C, Burch VC, et al. Warning scores in triageIs there any point? Afr J Emergency Med. 2012;2(3): 103-7. https://doi.org/10.1016/j.afjem.2012.04.004

7. Dalwai MK, Twomey M, Maikere J, et al. Reliability and accuracy of the South African Triage Scale when used by nurses in the emergency department of Timergara Hospital, Pakistan. S Afr Med J. 2014;104(5): 372-5. https://doi.org/10.7196/SAMJ.7604

8. Augustyn J. The South African Triage Scale: a tool for emergency nurses. Prof Nursing Today. 2011;15(6): 24-9.

9. Twomey M, de Sá A, Wallis LA, et al. Inter-rater reliability of the South AfricanTriageScale:Assessing twodifferentcadresofhealthcareworkers in a real time environment. Afr J Emergency Med. 2011;1(3): 113-118. https://doi.org/10.1016/j.afjem.2011.08.003

10. Twomey M, Wallis LA, Thompson ML, et al. The South African Triage Scale (adult version) provides reliable acuity ratings. Int Emerg Nurs. 2012;20(3): 142-150. https://doi.org/10.1016/j.ienj.2011.08.002

11. Bruijns SR, Wallis LA, Burch VC. Effect of introduction of nurse triage on waiting times in a South African emergency department. Emerg Med J. 2008;25(7): 395-397. https://doi.org/10.1136/emj.2007.049411
12. Rominski S, Bell SA, Oduro G, et al. The implementation of the South African Triage Score (SATS) in an urban teaching hospital, Ghana. Afr J Emergency Med. 2014;4(2): 71-75. https://doi.org/10.1016/j. afjem.2013.11.001

13. Naidoo D, Rangiah S, Naidoo S. An evaluation of the Triage Early Warning Score in an urban accident and emergency department in KwaZulu-Natal. South Afr Family Practice. 2014;56(1): 69-73. https://doi.org/10.1080/20786204.2014.10844586

14. Hanewinckel R, Jongman HP, Wallis LA, et al. Emergency medicine in Paarl, South Africa: a cross-sectional descriptive study. Int J Emerg Med. 2010;3(3): 143-50. https://doi.org/10.1007/s12245-010-0185-9

15. KobusingyeOC,GuwatuddeD,OworG,etal.Citywidetraumaexperience in Kampala, Uganda: a call for intervention. Inj Prev. 2002;8(2): 133-6. https://doi.org/10.1136/ip.8.2.133

16. Becker J, Dell A, Jenkins L, et al. Reasons why patients with primary health care problems access a secondary hospital emergency centre. S Afr Med J. 2012;102(10): 800-1. https://doi.org/10.7196/SAMJ.6059

17. Hodkinson PW, Wallis LA. Cross-sectional survey of patients presenting to a South African urban emergency centre. Emerg Med J. 2009;26(9): 635-40. https://doi.org/10.1136/emj.2008.063362

18. National Committee For Confidential Inquiry Into Maternal Deaths. Saving mother 2011-2013:Sixth report on the confidential enquiries into maternal deaths in South Africa Pretoria, South Africa. 2015.

19. Hoff WS, Tinkoff GH, Lucke JF, et al. Impact of minimal injuries on a level I trauma center. J Trauma. 1992;33(3): 408-412. https://doi.org/10.1097/00005373-199209000-00012

20. Rauf W, Blitz JJ, Geyser MM, et al. Quality improvement cycles that reduced waiting times at Tshwane District Hospital Emergency Department. South Afr Family Practice. 2008;50(6):43-e.

21. Pepper MS, Slabbert MN. Is South Africa on the verge of a medical malpractice litigation storm? South Afr J Bioethics and Law. 2011;4(1): 29-35.

Received: 03-11-2016 Accepted: 12-03-2017 